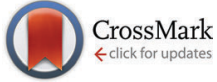

Cite this: Phys. Chem. Chem. Phys., 2015, 17, 29969

Received 10th September 2015 Accepted 7th October 2015

DOI: $10.1039 / c 5 c p 05400 a$

www.rsc.org/pccp

\title{
Single water solvation dynamics in the 4-aminobenzonitrile-water cluster cation revealed by picosecond time-resolved infrared spectroscopy
}

\author{
Mitsuhiko Miyazaki, ${ }^{a}$ Takashi Nakamura, ${ }^{a}$ Matthias Wohlgemuth, ${ }^{\mathrm{b}}$ Roland Mitrić, ${ }^{\mathrm{b}}$ \\ Otto Dopfer*c and Masaaki Fujii*a
}

\begin{abstract}
The dynamics of a solvent is important for many chemical and biological processes. Here, the migration dynamics of a single water molecule is triggered by the photoionization of the 4-aminobenzonitrile-water (4ABN-W) cluster and monitored in real time by picosecond time-resolved IR (ps TRIR) spectroscopy. In the neutral cluster, water is hydrogen-bonded to the $\mathrm{CN}$ group. When this $\mathrm{CN}$-bound cluster is selectively ionized with an excess energy of $1238 \mathrm{~cm}^{-1}$, water migrates with a lifetime of $\tau=17$ ps from the $\mathrm{CN}$ to the $\mathrm{NH}_{2}$ group, forming a more stable $4 A B N^{+}-W(N H)$ isomer with a yield of unity. By decreasing the ionization excess energy, the yield of the $\mathrm{CN} \rightarrow \mathrm{NH}_{2}$ reaction is reduced. The relatively slow migration in comparison to the ionization-induced solvent dynamics in the related acetanilide-water cluster cation ( $\tau=5 \mathrm{ps}$ ) is discussed in terms of the internal excess energy after photoionization and the shape of the potential energy surface.
\end{abstract}

\section{Introduction}

Solvent-solute interactions are some of the most important key factors in solution chemistry. ${ }^{1-7}$ They significantly affect the energetics and dynamics of chemical reactions and structural rearrangements, for example protein folding ${ }^{8-10}$ and the selfassembly of supramolecules and nanostructures. ${ }^{11-15}$ Particularly, the rearrangement of the solvent network, the solvation dynamics, is the initial process in solution-phase chemistry. One of the typical methods to observe the solvation dynamics has been the dynamic Stokes shift, i.e. the time-dependent spectral shift of fluorescence., Here, the photoexcitation of a solute molecule causes a sudden change in the solute-solvent interaction, which is the driving force for the rearrangement. The triggered gradual reorientation of the solvent induces a spectral shift in the fluorescence of the solute molecule. This method has been applied in time-resolved studies to a wide variety of systems, ranging from dyes ${ }^{6,16}$ to biological molecules, such as aromatic amino acid residues and proteins. $^{1,17,18}$ Although the dynamic Stokes shift provides a comprehensive view of the solvent rearrangement, it is difficult

\footnotetext{
${ }^{a}$ Chemical Resources Laboratory, Tokyo Institute of Technology, Yokohama 226-8503, Japan. E-mail: mfujii@res.titech.ac.jp

${ }^{b}$ Institut für Theoretische und Physikalische Chemie, Julius-Maximilians-Universität Würzburg, 97070 Würzburg, Germany. E-mail: roland.mitric@uni-wuerzburg.de ${ }^{c}$ Institut für Optik und Atomare Physik, Technische Universität Berlin, 10623 Berlin, Germany.E-mail: dopfer@physik.tu-berlin.de
}

to probe the dynamics of individual solvent molecules. On the other hand, recent progress of theoretical chemistry and rapid development of computer technology have provided realistic simulations of the dynamics of individual solvent molecules. Therefore, the experimental observation of solvation dynamics at a single molecular level is an essential demand to calibrate the up-to-date theoretical approach for the subsequent reliable prediction to problems in solution chemistry and biology.

Single molecular solvation dynamics can experimentally be studied in solvated clusters of solute and solvent molecules generated in molecular beams and stabilized by hydrogen bonds (H-bonds) and van der Waals interactions ( $\pi$ stacking). ${ }^{19-22}$ The solvated clusters are often considered as a nanodroplet or a local structure in solution. The first advantage of solvated clusters is that the number of solute and solvent molecules and their relative orientation can precisely be specified and controlled by combining mass spectrometry with resonant enhanced multiphoton ionization (REMPI). The second advantage is that the relative orientation of the molecules in the cluster is frozen because of efficient cooling in a supersonic expansion down to a few $\mathrm{K}$. The specific orientation can be controlled and precisely be determined by measuring, for example, their IR spectra and analysing them with quantum chemical calculations. This combined strategy of structural determination by comparison of observed and calculated IR spectra is well established for molecular clusters. ${ }^{23-26}$

To observe the solvation dynamics of aromatic clusters generated in molecular beams, we have developed the technique 
of mass-selective three-color tuneable UV-UV'-IR picosecond timeresolved infrared (ps TRIR) pump-probe laser spectroscopy. ${ }^{21,27-33}$ The different isomers of the solvated aromatic cluster can easily be distinguished by their unique $S_{1}-S_{0}$ UV transition energy. Then, a solvated cluster with a specific conformation can selectively be ionized by the REMPI process via $\mathrm{S}_{1}$ using two-color two-photon (UV-UV') ionization with adjustable ionization excess energy. In most cases, the sudden charge formation by photoionization causes a significant structural reorientation in the cluster, because the most stable structure of the charged cluster is usually different from that of the neutral reactant. The evolving structural dynamics can be traced in real time by measuring the IR spectra by a depletion technique at a variable delay time after the ionization event. In contrast to static spectroscopy, which only can detect the initial and final state of the reaction, such a dynamical approach yields additional essential information about the reaction (e.g. rate constant, reaction pathways, intermediates), which completely escapes static nanosecond spectroscopy.

Initially, this ps TRIR spectroscopic technique has been used to characterize the ionization-induced $\pi \rightarrow \mathrm{H}$ site-switching dynamics of a variety of rare gas ligands attached to the phenol molecule. ${ }^{21,28-30,32}$ Subsequently, this approach has been applied to the $\mathrm{CO} \rightarrow \mathrm{NH}$ site switching reaction in the transacetanilide-water cluster (AA-W), ${ }^{27}$ which is one of the simplest models of a hydrated peptide linkage (-CONH-). The ps TRIR spectra clearly contain many important details of this fundamental water migration process from the $\mathrm{CO}$ to the $\mathrm{NH}$ site of this linkage, including the two competing reaction pathways and their rate constants, the occurrence of transient intermediates, the reaction yield and its relation to intracluster vibrational energy redistribution (IVR). ${ }^{27,34}$ This migration reaction and the resulting TRIR spectra were subsequently analysed and visualized by "on-the-fly" molecular dynamics (MD) simulations. ${ }^{34}$ The important aspect of this combined experimental and computational approach is that the MD simulations could precisely be calibrated by the accurate reproduction of the measured TRIR spectra. The experimentally calibrated simulations revealed that the water migration reaction with 5 ps duration has two pathways, namely a fast and a slow channel, and only the slow channel has an intermediate. This detailed visualization is the first example of combined experimental and theoretical characterization of single molecular solvation dynamics in clusters and in general. The combination of ps TRIR spectroscopy and MD simulations calibrated by experiment can now be applied to various other solvated clusters and therefore we have chosen the 4-aminobenzonitrile-water $(4 \mathrm{ABN}-\mathrm{W})$ cluster as the second example to be explored by this combination. ${ }^{35-44}$ Here, we present the first step of this combined strategy, namely the measurement of the ps TRIR spectra of $4 \mathrm{ABN}^{+}-\mathrm{W}$ and their preliminary analysis by a classical rate equation model.

Ionization-induced water migration has been suggested in several solvated clusters by static nanosecond IR laser spectroscopy of the neutral reactant and the final cation product, including monohydrated clusters of benzene, ${ }^{22,45-47}$ aminophenol, ${ }^{48}$ formanilide, ${ }^{49-51}$ acetanilide,$^{27,52,53}$ tryptamine ${ }^{54,55}$ phenylglycine, ${ }^{56}$ and $4 \mathrm{ABN} .{ }^{43,44}$ From these candidates, we have selected
$4 \mathrm{ABN}-\mathrm{W}$ for the following reasons. $4 \mathrm{ABN}$ is a benzene derivative substituted with an electron-donating $\mathrm{NH}_{2}$ group and an electronwithdrawing $\mathrm{CN}$ group. It provides several competing binding sites for water, and three different isomers of $4 \mathrm{ABN}-\mathrm{W}$ coexist in the $\mathrm{S}_{0}$ state, namely the $\mathrm{NH}$-bound $4 \mathrm{ABN}-\mathrm{W}(\mathrm{NH})$ isomer in which water is $\mathrm{H}$-bonded to the $\mathrm{NH}_{2}$ group and two CN-bound $4 \mathrm{ABN}-\mathrm{W}(\mathrm{CN})$ clusters, in which water is $\mathrm{H}$-bonded in either a bent or linear configuration to the CN group. ${ }^{39}$ The latter can be detected only by fluorescence spectroscopy and thus can be neglected in this work. $4 \mathrm{ABN}-\mathrm{W}(\mathrm{CN})$ holds its binding motif by excitation into $\mathrm{S}_{1}$, but shows the water migration from the $\mathrm{CN}$ to the $\mathrm{NH}_{2}$ site upon ionization. ${ }^{43}$ The large change in the charge distribution of the aromatic chromophore substantially destabilises the $\mathrm{CN}$ structure with respect to the $\mathrm{NH}$ isomer in $4 \mathrm{ABN}^{+}-\mathrm{W}$. This $\mathrm{CN} \rightarrow \mathrm{NH}_{2}$ site switching with $100 \%$ yield was inferred from the drastic change in the IR spectra before and after ionization but the dynamics of this water migration and other parameters of the reaction have not been characterized yet. To this end, we apply UV-UV'-IR ps TRIR spectroscopy to $4 \mathrm{ABN}-\mathrm{W}(\mathrm{CN})$ to monitor the water migration dynamics in real time by the time evolution of the IR spectra measured at a variable delay time $\Delta t$ after the ionization. Details of the $\mathrm{CN} \rightarrow \mathrm{NH}_{2}$ water migration reaction triggered by ionization of the $4 \mathrm{ABN}-\mathrm{W}(\mathrm{CN})$ isomer have been revealed. We also investigate the effect of the ionization excess energy on the reaction yield and discuss its relation to the shape of the potential energy surface and the reaction path in the cation ground state of $4 \mathrm{ABN}^{+}-\mathrm{W}$.

\section{Experimental}

The principle for the measurement of the ps time-resolved UV-UV'-IR ion dip (TRIR) spectra are shown in Fig. 1 and are described in detail elsewhere. ${ }^{21,32,33}$ Briefly, $4 \mathrm{ABN}-\mathrm{W}$ clusters are produced in a supersonic jet by expanding $4 \mathrm{ABN}$ vapour $\left(70{ }^{\circ} \mathrm{C}\right)$ and a trace amount of residual water seeded in He gas (3 bar) through a pulsed valve into a vacuum chamber. Neutral $4 \mathrm{ABN}-\mathrm{W}$ dimers are ionized from the neutral ground state $\left(\mathrm{S}_{0}\right)$ employing resonant two-color two-photon $\left(1+1^{\prime}\right)$ REMPI through the first excited singlet state $\left(\mathrm{S}_{1}\right)$ using two ps UV laser beams, $\nu_{\text {exc }}$ (UV) and $\nu_{\text {ion }}$ (UV'). $\nu_{\text {ion }}$ is irradiated at a delay time of 106 ps after $\nu_{\text {exc }}$ (corresponding to $\left.\Delta t=0\right) . \nu_{\text {exc }}$ and $\nu_{\text {ion }}$ are tuned to $33678\left(\mathrm{~S}_{1} 0^{0}\right)$ and $32780 \mathrm{~cm}^{-1}$ for resonant ionization of the $4 \mathrm{ABN}-\mathrm{W}(\mathrm{CN})$ isomer, respectively. ${ }^{36,37,39-41,57-59}$ Thus, according to the reported photoionization efficiency curve, the maximum internal energy of generated $4 \mathrm{ABN}^{+}-\mathrm{W}(\mathrm{CN})$ cation is $1238 \mathrm{~cm}^{-1}$ with respect to its ionization energy of $65220 \mathrm{~cm}^{-1}$. The ions generated in the cation ground state $\left(D_{0}\right)$ are extracted into a quadrupole mass spectrometer. While monitoring the mass-selected $4 \mathrm{ABN}^{+}-\mathrm{W}$ signal, a tuneable ps IR laser, $\nu_{\mathrm{IR}}$, is fired at a delay time of $106 \mathrm{ps}(\Delta t=0)$ after $\nu_{\text {exc }}$ and scanned through the $2800-3800 \mathrm{~cm}^{-1}$ vibrational range at an adjustable delay $(\Delta t)$ with respect to $\nu_{\text {ion }}$. The ion signal is amplified, integrated, and monitored as a function of $\nu_{\mathrm{IR}}$ and/or $\Delta t$. When $\nu_{\mathrm{IR}}$ is resonant with a vibrational transition of $4 \mathrm{ABN}^{+}-\mathrm{W}$, the cluster dissociates upon vibrational excitation, causing a depletion (dip) in the parent ion current. Thus, the IR spectrum of 


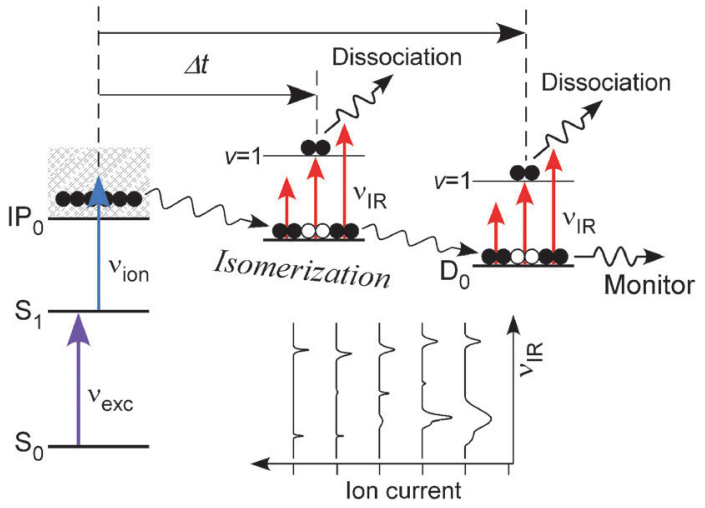

Fig. 1 Strategy of picosecond time-resolved IR ion dip (ps TRIR) spectroscopy. $4 A B N-W(C N)$ is selectively excited $\left(\nu_{\text {exc }}\right)$ and ionized $\left(\nu_{\text {ion }}\right)$ by two ps UV laser beams ( $1+1^{\prime}$ REMPI) to trigger the water migration reaction. The dynamics of this reaction is monitored by a ps IR beam $\left(\nu_{\mid \mathrm{R}}\right)$ delayed by $\Delta t$ from $\nu_{\text {ion. }}$. The IR absorption dissociates the cluster and the resulting depletion of the parent ion signal gives its IR spectrum. If the frequency of the IR laser is fixed to a specific vibrational IR transition and $\Delta t$ is scanned, the time evolution of this IR transition is obtained. The time profile represents the population change of the species with this characteristic IR transition.

$4 \mathrm{ABN}^{+}-\mathrm{W}$ is obtained by monitoring the depletion of the ion current as a function of $\nu_{\mathrm{IR}}$. To monitor the population change of $4 \mathrm{ABN}^{+}-\mathrm{W}$ associated with a particular vibrational transition, $\nu_{\mathrm{IR}}$ is fixed to this resonance and $\Delta t$ is varied by scanning the delay stage for $\nu_{\text {ion }}$ to shorter time with respect to $\nu_{\text {exc }}$. The time evolution of the signal depletion is converted to the population change by taking the logarithm of the depletion. The generation of the ps UV/UV' and IR laser pulses is described elsewhere. $^{21,32,33}$ The key parameters are a $10 \mathrm{~Hz}$ repetition rate, 3 ps pulse widths, $12 \mathrm{~cm}^{-1}$ spectral resolution, and pulse energies of $1 \mu \mathrm{J}, 10 \mu \mathrm{J}$, and $50-70 \mu \mathrm{J}$ for $\nu_{\mathrm{exc}}, \nu_{\mathrm{ion}}$, and $\nu_{\mathrm{IR}}$, respectively. The UV/UV' and IR laser beams are combined coaxially and focused by a $\mathrm{CaF}_{2}$ lens with $300 \mathrm{~mm}$ focal length into the supersonic expansion. Similar REMPI and IR depletion experiments have also been performed using standard IR/UV nanosecond lasers, as described in detail previously. ${ }^{60}$

\section{Results and discussion}

\subsection{REMPI spectrum}

Fig. 2(a) and (b) show the $1+1^{\prime}$ REMPI and $1+1$ REMPI spectra of $4 \mathrm{ABN}-\mathrm{W}$ measured using picosecond and nanosecond lasers, respectively. The nanosecond spectrum well reproduces previous reports. ${ }^{36,37,39-41,57-59}$ Bands at 32950 and $33678 \mathrm{~cm}^{-1}$ have been assigned to $\mathrm{S}_{1} 0^{0}$ bands of the $\mathrm{NH}$ and $\mathrm{CN}$ isomers, respectively. The other peaks are ascribed to vibronic transitions originating from zero-point vibrational levels in the $S_{0}$ state of these isomers, ${ }^{39,43}$ and no hot band transition is observed. This result confirms that the internal (vibrational) degrees of freedom of the clusters are efficiently cooled down in the jet. The $1+1^{\prime}$ REMPI spectrum obtained by picosecond lasers reproduces well that recorded by nanosecond lasers, although the widths of the bands are broadened due to the inferior spectral resolution of

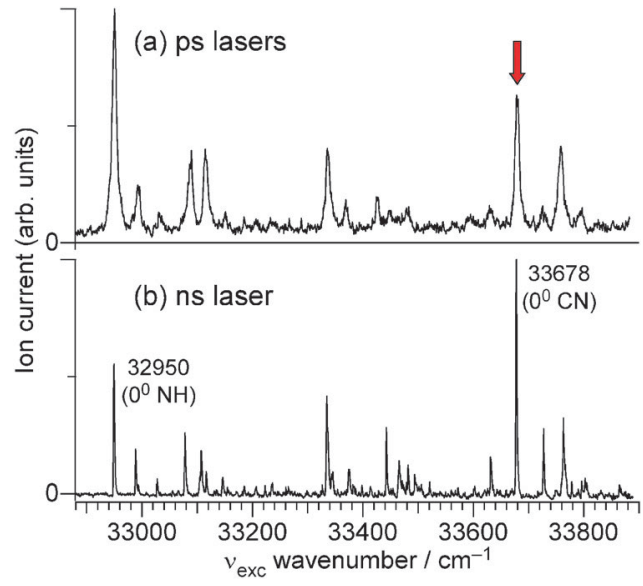

Fig. 2 REMPI spectra of $4 A B N-W$ recorded using ps lasers $\left(a, 1+1^{\prime}\right.$ REMPI) and a ns laser (b, $1+1$ REMPI). In the ps time-resolved experiments, $\nu_{\text {exc }}$ is fixed to the $\mathrm{S}_{1} \mathrm{O}^{\circ}$ band of the $\mathrm{ABN}-\mathrm{W}(\mathrm{CN})$ isomer indicated by the arrow.

the picosecond laser pulses $\left(12 \mathrm{~cm}^{-1}\right)$. The $\mathrm{S}_{1} 0^{0}$ band of each cluster is still well resolved from the other transitions, ensuring selective excitation of the $\mathrm{CN}$ isomer to its $\mathrm{S}_{1} 0^{0}$ origin under these conditions, as also confirmed by the previously reported hole-burning spectra. ${ }^{39,43}$ Thus, the time-resolved spectroscopy is carried out by fixing $\nu_{\text {exc }}$ to the $\mathrm{S}_{1} 0^{0}$ band of the $\mathrm{CN}$ isomer.

\subsection{Picosecond time-resolved IR spectra}

Fig. 3(c) shows the ps TRIR spectra measured by varying $\Delta t$ from -3 to $+59 \mathrm{ps}$. For comparison, static nanosecond IR spectra of $4 \mathrm{ABN}^{+}-\operatorname{Ar}(\pi), 4 \mathrm{ABN}-\mathrm{W}(\mathrm{CN})$ (reactant $\mathrm{R}$ ) in the $\mathrm{S}_{1}$ state and $4 \mathrm{ABN}^{+}-\mathrm{W}(\mathrm{NH})$ corresponding to the reaction product $\left(\mathrm{P}^{+}, \Delta t=+50 \mathrm{~ns}\right)$ measured by nanosecond lasers are shown in Fig. 3(a), (b) and (d), respectively. ${ }^{43,59}$ In this excitation scheme, the IR absorption of $4 \mathrm{ABN}-\mathrm{W}(\mathrm{CN})$ in $\mathrm{S}_{1}$ overlaps with the IR absorption of the nascent $4 \mathrm{ABN}^{+}-\mathrm{W}$ clusters in the ps TRIR spectra of $\mathrm{D}_{0}$ at small delay because of the temporal overlap of the $\nu_{\text {ion }}$ and $\nu_{\text {IR }}$ pulses ( $\sim 4$ ps from a cross-correlation measurement). Specifically, a sharp band B at $3409 \mathrm{~cm}^{-1}$ colored in red in the nanosecond spectrum of the $S_{1}$ state of $4 \mathrm{ABN}-\mathrm{W}(\mathrm{CN})$ is assigned to the free $\mathrm{NH}_{2}$ symmetric stretching vibration $\left(\nu_{\mathrm{NH}}^{\mathrm{S}}\left(\mathrm{S}_{1}\right)\right)$. The corresponding picosecond TRIR spectra before photoionization $(\Delta t<0)$ show the same $\nu_{\mathrm{NH}}^{\mathrm{s}}\left(\mathrm{S}_{1}\right)$ transition. The antisymmetric stretching mode of free $\mathrm{NH}_{2}$ is also observed at $3526 \mathrm{~cm}^{-1}$ in the nanosecond spectrum but not detected in the ps TRIR spectra because of its weak intensity. The bands in the picosecond TRIR spectra are broader because of the lower spectral resolution $\left(12 \mathrm{~cm}^{-1}\right)$ arising from the shorter laser pulse duration. The ps TRIR spectra change immediately after ionization $(\Delta t=1$ and $2 \mathrm{ps})$. The $\nu_{\mathrm{NH}}^{\mathrm{s}}\left(\mathrm{S}_{1}\right)$ band $\mathrm{B}$ of the reactant $\mathrm{R}$ becomes weaker and disappears after around $\Delta t=2 \mathrm{ps}$ (consistent with the pulse duration of the ps laser system). Instead, the TRIR spectrum at $\Delta t=0$ shows a new band A at $3390 \mathrm{~cm}^{-1}$ (green) assigned to the $\mathrm{NH}_{2}$ free symmetric stretching mode of $4 \mathrm{ABN}^{+}\left(\nu_{\mathrm{NH}}^{\mathrm{S}}\right)$, as inferred from the experimental $4 \mathrm{ABN}^{+}-\mathrm{Ar}$ spectrum in Fig. 3(a). ${ }^{59,61}$ This band A can be seen in the TRIR 


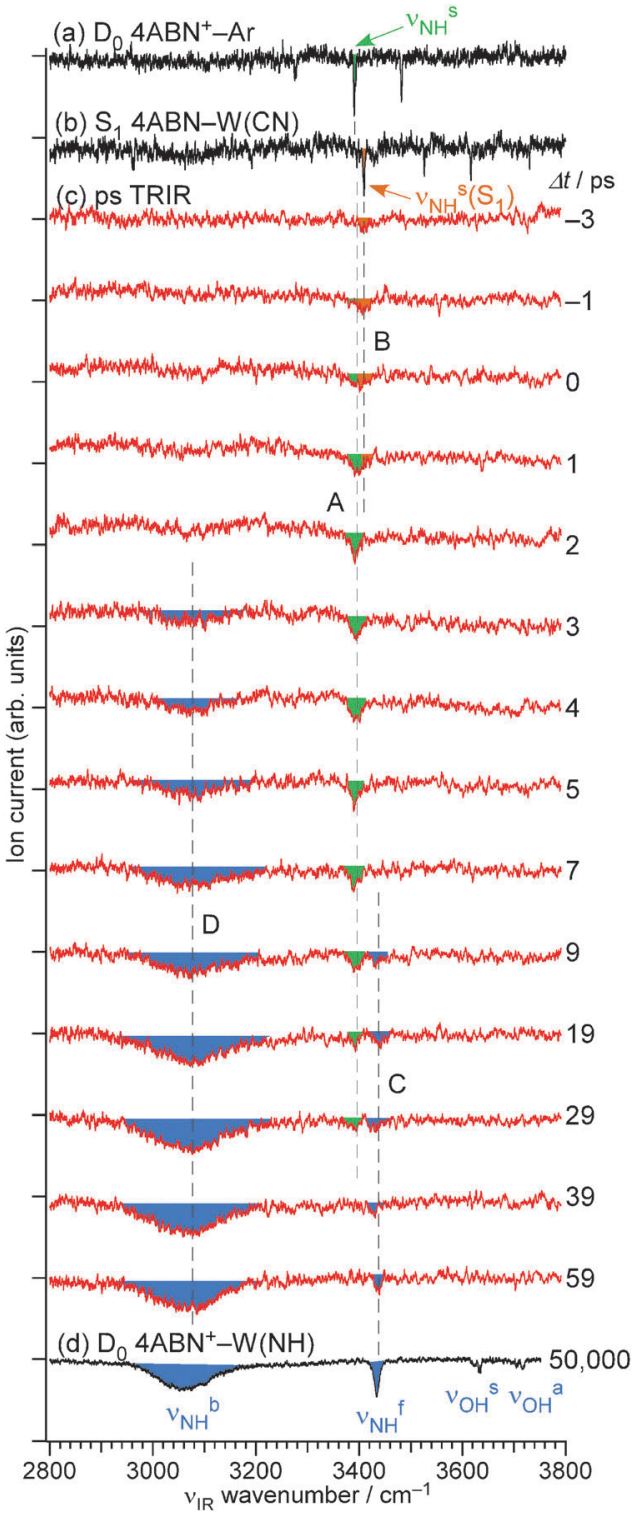

Fig. 3 Picosecond time-resolved IR ion dip (ps TRIR) spectra of $4 A B N^{+}-W$ for selective ionization of the $4 A B N-W(C N)$ isomer via $1+1^{\prime}$ REMPI as a function of $\Delta t\left(E_{\text {exc }}=1238^{-1}\right)$ (c). Static spectra measured for the $\mathrm{S}_{1}$ state (b) and the $\mathrm{D}_{0}$ state (d) using nanosecond lasers are shown for comparison. ${ }^{43}$ The IR spectrum of cold $4 \mathrm{ABN}^{+}-\mathrm{Ar}$ clusters is shown in (a). ${ }^{59}$ The positions used to monitor time evolutions (bands $\mathrm{A}, \mathrm{C}$ and $\mathrm{D}$ ) are indicated by dashed lines.

spectra at $\Delta t=0$ and 1 ps adjacent to the $\nu_{\mathrm{NH}}^{\mathrm{s}}\left(\mathrm{S}_{1}\right)$ band B. Its intensity gradually increases, decays again after around $\Delta t=9 \mathrm{ps}$, and has completely disappeared before $39 \mathrm{ps}$. Before $\Delta t=3 \mathrm{ps}$, no other intense band is observed, in particular in the range below $3300 \mathrm{~cm}^{-1}$. Thus, the water ligand has to be either located still at the $\mathrm{CN}$ site and/or has already been released from the $\mathrm{CN}$ site but has not yet arrived at the $\mathrm{NH}_{2}$ site.

After $\Delta t=3 \mathrm{ps}$, a new broad band D (blue) at around $3080 \mathrm{~cm}^{-1}$ appears and grows with increasing delay. In addition, a much narrower band $\mathrm{C}$ (blue) at $3434 \mathrm{~cm}^{-1}$ appears after $\Delta t=7 \mathrm{ps}$. Finally, the spectrum at $\Delta t=39 \mathrm{ps}$ has converged and resembles the static one observed at $\Delta t=50 \mathrm{~ns}$ after ionization recorded by nanosecond lasers. These bands $\mathrm{D}$ and $\mathrm{C}$ were assigned to the $\mathrm{H}$-bonded and free NH stretching vibrations, $\nu_{\mathrm{NH}}^{\mathrm{b}}$ and $\nu_{\mathrm{NH}}^{\mathrm{f}}$, of the amino group, respectively, arising from $\mathrm{H}$-bonding with water. ${ }^{43}$ Those are the spectral signatures characteristic of the final $4 \mathrm{ABN}^{+}-\mathrm{W}(\mathrm{NH})$ reaction product, $\mathrm{P}^{+}{ }^{43,44}$ Therefore, the disappearance of $\nu_{\mathrm{NH}}^{\mathrm{s}}$ and the subsequent appearance of $\nu_{\mathrm{NH}}^{\mathrm{b}}$ and $\nu_{\mathrm{NH}}^{\mathrm{f}}$ unambiguously demonstrate the direct time-resolved observation of the water migration from the $\mathrm{CN}$ to the $\mathrm{NH}_{2}$ site of $4 \mathrm{ABN}^{+}$ triggered by photoionization of $4 \mathrm{ABN}^{+}-\mathrm{W}(\mathrm{CN})$. The symmetric and antisymmetric $\mathrm{OH}$ stretching vibrations of the water ligand $\left(\nu_{\mathrm{OH}}^{\mathrm{s}}\right.$ and $\nu_{\mathrm{OH}}^{\mathrm{a}}$, respectively) cannot be detected in the ps TRIR spectra although both bands are clearly observed in the nanosecond spectrum at $\Delta t=50 \mathrm{~ns}$ as sharp and weak bands. The energy resolution of the picosecond IR laser is lower, and thus such sharp and weak transitions cannot be detected in the ps TRIR spectra under the current sensitivity conditions.

\subsection{Time evolution of IR absorption}

The ps TRIR spectra probe the water migration from the $\mathrm{CN}$ to the $\mathrm{NH}_{2}$ binding site triggered by photoionization in real time. In this dynamical process, we should consider the populations of the reactant $\mathrm{R}\left(4 \mathrm{ABN}-\mathrm{W}(\mathrm{CN})\right.$ in $\left.\mathrm{S}_{1}\right)$, the Franck-Condon state $\mathrm{FC}^{+}\left(4 \mathrm{ABN}^{+}-\mathrm{W}(\mathrm{CN})\right)$ prepared by vertical ionization of $\mathrm{R}$, and the product $\mathrm{P}^{+}\left(4 \mathrm{ABN}^{+}-\mathrm{W}(\mathrm{NH})\right)$. In the water migration dynamics of the $\mathrm{AA}^{+}-\mathrm{W}$ cation, the water ligand migrates from the initially CO-bound site to the final NH-bound site via an intermediate $\mathrm{I}^{+}$, in which the water ligand does bind neither at the initial nor final binding site. Thus, we may also consider the existence of such an $\mathrm{I}^{+}$state for the $\mathrm{CN} \rightarrow \mathrm{NH}_{2}$ migration reaction in $4 \mathrm{ABN}^{+}-\mathrm{W}$. However, the ps TRIR spectra of $4 \mathrm{ABN}^{+}-\mathrm{W}$ do not detect the $\mathrm{H}$-bonded $\mathrm{OH}$ stretching vibration of the water ligand, and as a result, $\nu_{\mathrm{NH}}^{\mathrm{S}}$ is the only spectral signature of $\mathrm{FC}^{+}$. Unfortunately, $\nu_{\mathrm{NH}}^{\mathrm{S}}$ is also characteristic of any $\mathrm{I}^{+}$candidate. Hence, we cannot separate $\mathrm{FC}^{+}$from any intermediate $\mathrm{I}^{+}$in the measured ps TRIR spectra, even if such an $\mathrm{I}^{+}$ were to exist. Consequently, the time-evolution of $\nu_{\mathrm{NH}}^{\mathrm{s}}$ corresponds to the sum of the populations of $\mathrm{FC}^{+}$and possibly $\mathrm{I}^{+}$in this analysis, denoted $\mathrm{AF}^{+}$(amino-free species), i.e., the population of ions with a free $\mathrm{NH}_{2}$ group.

As already deduced from the nanosecond experiments, the ps TRIR spectra in Fig. 3(c) clearly confirm that essentially all ionized CN-bound clusters are converted to NH-bound clusters with $100 \%$ yield at the employed ionization excess energy of $1238 \mathrm{~cm}^{-1}$. As the clusters are isolated systems without the possibility of energy release, the $\mathrm{NH}_{2} \rightarrow \mathrm{CN}$ back reaction may be expected and has indeed been observed for the $\pi \leftrightarrow H$ reaction in the phenol ${ }^{+}-\mathrm{Kr}$ dimer. ${ }^{21,28}$ In this cluster, the $\mathrm{Kr}$ atom migrates from the initial $\pi$ binding site above the aromatic ring to $\mathrm{H}$-bonded $\mathrm{OH}$ site upon ionization. However the $\mathrm{H} \rightarrow \pi$ back reaction eventually causes a nonvanishing $\pi \leftrightarrow \mathrm{H}$ equilibrium. For $4 \mathrm{ABN}^{+}-\mathrm{W}$, the lack of any $\mathrm{NH}_{2} \rightarrow \mathrm{CN}$ back reaction and unity yield for the $\mathrm{CN} \rightarrow \mathrm{NH}_{2}$ forward reaction indicates that efficient IVR in $\mathrm{P}^{+}$prevents the back reaction because of fast removal of vibrational energy from the reaction coordinate. The efficient IVR is attributed to the bath modes arising from the intermolecular vibrations not involved in the reaction coordinate 
and intramolecular modes below $\sim 3500 \mathrm{~cm}^{-1}$, resulting from the sum of the exothermicity of the $\mathrm{CN} \rightarrow \mathrm{NH}_{2}$ reaction $\left(2332 \mathrm{~cm}^{-1}\right)$ and the ionization excess energy of $1238 \mathrm{~cm}^{-1}$ used in the two-color ps experiments. ${ }^{44}$

The time evolutions describing the $\mathrm{CN} \rightarrow \mathrm{NH}_{2}$ forward reaction measured at three relevant vibrational bands, namely $\nu_{\mathrm{NH}}^{\mathrm{s}}\left(\right.$ band A), $\nu_{\mathrm{NH}}^{\mathrm{f}}$ (band $\left.\mathrm{C}\right)$, and $\nu_{\mathrm{NH}}^{\mathrm{b}}$ (band D), probed at dashed lines in Fig. 3 are shown in Fig. 4(a)-(c), respectively. Here, $\nu_{\mathrm{NH}}^{\mathrm{S}}$ corresponds to $\mathrm{AF}^{+}\left(\mathrm{FC}^{+}+\mathrm{I}^{+}\right)$while the latter two bands represent $\mathrm{P}^{+}$. Although only two bands corresponding to $\mathrm{AF}^{+}$ and $\mathrm{P}^{+}$are observed in the TRIR spectra, $\mathrm{FC}^{+}$and $\mathrm{I}^{+}$are treated separately from $\mathrm{AF}^{+}$because the inclusion of $\mathrm{I}^{+}$in the rate equations usually results in a multi-exponential behaviour even for $\left[\mathrm{AF}^{+}\right]=\left[\mathrm{FC}^{+}+\mathrm{I}^{+}\right]$. Although various reaction pathways are feasible, we consider the simplest case, namely the onedirectional two-step forward reaction, $\mathrm{FC}^{+} \rightarrow \mathrm{I}^{+} \rightarrow \mathrm{P}^{+}$in our initial analysis. The rate equations for this reaction scheme are expressed as follows:

$$
\begin{gathered}
\frac{\mathrm{d}\left[\mathrm{FC}^{+}\right]}{\mathrm{d} t}=-k_{1}\left[\mathrm{FC}^{+}\right], \\
\frac{\mathrm{d}\left[\mathrm{I}^{+}\right]}{\mathrm{d} t}=k_{1}\left[\mathrm{FC}^{+}\right]-k_{2}\left[\mathrm{I}^{+}\right], \\
\frac{\mathrm{d}\left[\mathrm{P}^{+}\right]}{\mathrm{d} t}=k_{2}\left[\mathrm{I}^{+}\right] .
\end{gathered}
$$

Here, $[X]$ are the populations of $X$, and $k_{1}$ and $k_{2}$ are rate constants. The rate eqn (1)-(3) are solved under an initial condition of $\left[\mathrm{FC}^{+}\right](t=0)=1,\left[\mathrm{I}^{+}\right](t=0)=\left[\mathrm{P}^{+}\right](t=0)=0$ :

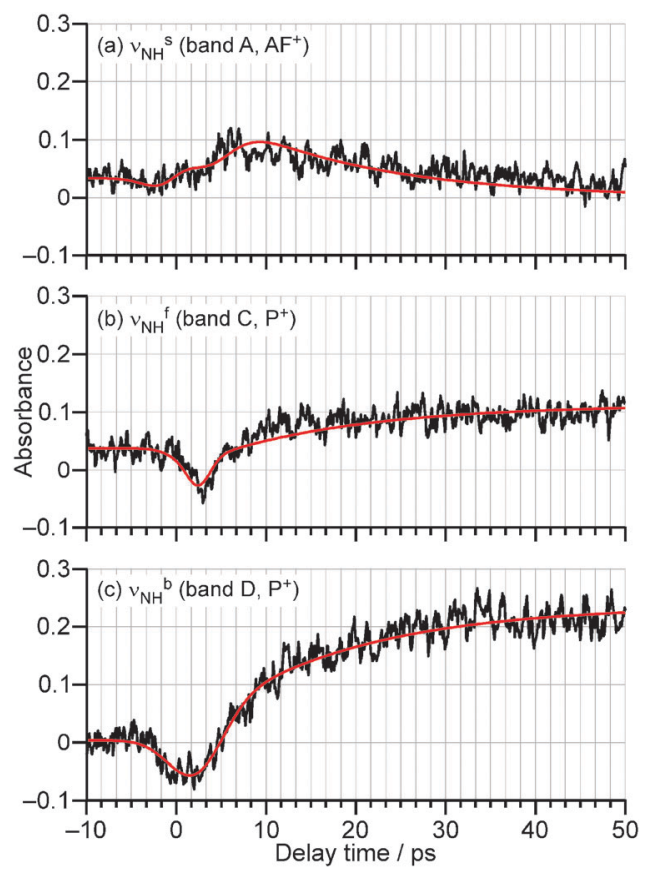

Fig. 4 Experimental time evolution of three vibrational bands ( $A, C$ and $D$ ) of $4 \mathrm{ABN}^{+}-\mathrm{W}$ as indicated in Fig. 3: (a) $\nu_{\mathrm{NH}}^{\mathrm{s}}$ (band $\mathrm{A}$, green, $\mathrm{AF}^{+}$); (b) $\nu_{\mathrm{NH}}^{\mathrm{f}}$ (band $C$, blue, $\mathrm{P}^{+}$); (c) $\nu_{\mathrm{NH}}^{\mathrm{b}}\left(\right.$ band $\mathrm{D}$, blue, $\mathrm{P}^{+}$). The best fit curves obtained by a two-step reaction model are included in red.

$$
\begin{gathered}
{\left[\mathrm{FC}^{+}\right]=\mathrm{e}^{-k_{1} t},} \\
{\left[\mathrm{I}^{+}\right]=\frac{k_{1}}{k_{2}-k_{1}}\left(\mathrm{e}^{-k_{1} t}-\mathrm{e}^{-k_{2} t}\right),} \\
{\left[\mathrm{P}^{+}\right]=\frac{1}{k_{2}-k_{1}}\left\{k_{2}\left(1-\mathrm{e}^{-k_{1} t}\right)-k_{1}\left(1-\mathrm{e}^{-k_{2} t}\right)\right\},}
\end{gathered}
$$

Thus, the total population change of $\mathrm{FC}^{+}$and $\mathrm{I}^{+}$corresponding to the time evolution of free $\nu_{\mathrm{NH}}^{\mathrm{s}}$ is:

$$
\begin{aligned}
{\left[\mathrm{FC}^{+}+\mathrm{I}^{+}\right] } & =\left[\mathrm{AF}^{+}\right]=\frac{1}{k_{2}-k_{1}}\left(k_{2} \mathrm{e}^{-k_{1} t}-k_{1} \mathrm{e}^{-k_{2} t}\right) \\
& =1-\left[\mathrm{P}^{+}\right] .
\end{aligned}
$$

These results are multiplied by an unit step function at $t=0$, $u(t)$, to express the sudden production of $\mathrm{FC}^{+}$at $t=0$. In addition, the cross-correlation between $\nu_{\text {ion }}$ and $\nu_{\mathrm{IR}}$, which can be approximated by a Gaussian profile with 4 ps FWHM, is taken into account as a Gaussian convolution on these expressions.

Final fits to the experimental time profiles (red traces in Fig. 4) yield $1 / k_{1}=\tau_{1} \leq 0.1 \mathrm{ps}$ and $1 / k_{2}=\tau_{2}=17 \pm 2 \mathrm{ps}$. The obtained fits reasonably well reproduce the experimental time evolutions. Nevertheless, due to coherent spikes near $\Delta t=0$, which are taken empirically into account by Gaussian functions in the fitting, a definite analysis near $\Delta t=0$ is difficult. Moreover, because expressions (5)-(7) are symmetric with respect to $k_{1}$ and $k_{2}$, interconversion of $k_{1}$ and $k_{2}$ gives the same trace. Therefore, from the observed data one cannot determine the ratedetermining step, unless the decay rate of $\left[\mathrm{FC}^{+}\right]$is monitored independently. In addition, as mentioned below, the cationic potential energy surface has several local shallow minima with similar depths. Thus, both steps could be the rate-determining one. Moreover, more complicated reaction schemes evolve if these local minima are taken into account. Hence, more experimental data and/or sophisticated MD simulations are required to fully analyse the $\mathrm{CN} \rightarrow \mathrm{NH}_{2}$ reaction mechanism.

Interestingly, the total reaction time of $\sim 17$ ps derived for the $\mathrm{CN} \rightarrow \mathrm{NH}_{2}$ isomerization in $4 \mathrm{ABN}^{+}-\mathrm{W}$ from the simple rate equation analysis is much longer than that of the $\mathrm{CO} \rightarrow \mathrm{NH}$ water migration in $\mathrm{AA}^{+}-\mathrm{W}$, which takes only $5 \mathrm{ps}^{27,34}$ As both reactions are water migrations around simple benzene derivatives, there is not a substantial difference in the length of the reaction paths. Then, the speed of the water migration in $4 \mathrm{ABN}^{+}-\mathrm{W}$ should be slower than that in $\mathrm{AA}^{+}-\mathrm{W}$. The difference of the reaction rate may be related to the different internal energies of the cluster cations. As mentioned above, $4 \mathrm{ABN}^{+}-\mathrm{W}(\mathrm{CN})$ is generated here with an excess energy of $1238 \mathrm{~cm}^{-1}$ by two-color REMPI, which corresponds to the maximum internal energy of the cation directly after ionization. On the other hand, in the previous study of $\mathrm{AA}^{+}-\mathrm{W}$, the $\mathrm{AA}^{+}-\mathrm{W}(\mathrm{CO})$ isomer was generated by one-color REMPI. The ionization energy of AA-W(CO) has not been determined yet, but it is lower than $64870 \mathrm{~cm}^{-1}$ derived from the twocolor REMPI spectrum. ${ }^{62}$ Based on this estimation, the excess energy of $\mathrm{AA}^{+}-\mathrm{W}$ after ionization is above $7230 \mathrm{~cm}^{-1}$. According to the theoretical calculation at the M06-2X/aug-cc-pVTZ level, the ionization potential of the reactant $\mathrm{AA}-\mathrm{W}(\mathrm{CO})$ is $2500 \mathrm{~cm}^{-1}$ above that of 
the product $\mathrm{AA}-\mathrm{W}(\mathrm{NH})$, and then the excess energy for ionization is about $6000 \mathrm{~cm}^{-1} .{ }^{63}$ Because of the Franck-Condon principle, this value is the maximum internal (mostly vibrational) energy of the generated cations, and usually not all the excess energy can be converted into internal energy (the remaining energy goes into kinetic energy of the photoelectron). However, although the Franck-Condon factors are not well known for both ionization processes, more than a factor five difference in the ionization excess energy may result in a significant difference in the initial internal energies of $\mathrm{AA}^{+}-\mathrm{W}(\mathrm{CO})$ and $4 \mathrm{ABN}^{+}-\mathrm{W}(\mathrm{CN})$. Thus, one of the reasons for the slower $\mathrm{CN} \rightarrow \mathrm{NH}_{2}$ isomerization of $4 \mathrm{ABN}^{+}-\mathrm{W}$ may be the smaller available internal energy. In addition, possible reaction barriers will also affect the rate constant. Density functional (DFT) calculations at the M06-2X/aug-cc-pVTZ level indeed exhibit a CN-bound local minimum of $4 \mathrm{ABN}^{+}-\mathrm{W}$ and a transition state near the minimum with a barrier of $377 \mathrm{~cm}^{-1}$ (Fig. 5) for isomerization toward the NH-bound global minimum along the in-plane path. ${ }^{44}$ However, also for $\mathrm{AA}^{+}-\mathrm{W}$ a barrier between the CO-bound and $\mathrm{NH}$-bound minima has been predicted. ${ }^{34}$ Hence, currently we attribute the faster rate in $\mathrm{AA}^{+}-\mathrm{W}$ to the larger excess energy. Future time-resolved experiments at various excess energies are required to explore the dependence of the reaction rate on the excess energy and the barrier heights.

\subsection{Reaction rate and excess energy}

To explore the effect of the ionization excess energy on the migration reaction, we consider the IR spectra after ionization of $4 \mathrm{ABN}-\mathrm{W}(\mathrm{CN})$ via its $\mathrm{S}_{1}$ origin with variable excess energy by using nanosecond laser systems. The excitation scheme is essentially the same as that in Fig. 1 but all picosecond lasers are replaced by tuneable nanosecond lasers. The IR spectra measured at $\Delta t=50 \mathrm{~ns}$ are shown in Fig. 6 for excess energies between 2136 and $296 \mathrm{~cm}^{-1}$. The spectra are measured in the vicinity of $\nu_{\mathrm{NH}}^{\mathrm{b}}$ (signature of $\left.\mathrm{P}^{+}\right), \nu_{\mathrm{NH}}^{\mathrm{s}}$ and $2 \delta_{\mathrm{NH}_{2}}\left(\mathrm{FC}^{+}\right.$and $\mathrm{I}^{+}$), and $\nu_{\mathrm{NH}}^{\mathrm{f}}\left(\mathrm{P}^{+}\right)$. At a relatively high ionization excess energy of $2136 \mathrm{~cm}^{-1}$ above ionization potential ( $\mathrm{IP}_{0}$ ) (Fig. 6(a), $1+1 \mathrm{REMPI}$ ), both $\nu_{\mathrm{NH}}^{\mathrm{b}}$ and $\nu_{\mathrm{NH}}^{\mathrm{f}}$ appear clearly but $\nu_{\mathrm{NH}}^{\mathrm{s}}$ cannot be seen, indicating a $\mathrm{CN} \rightarrow \mathrm{NH}_{2}$ reaction yield of close to unity. This result is

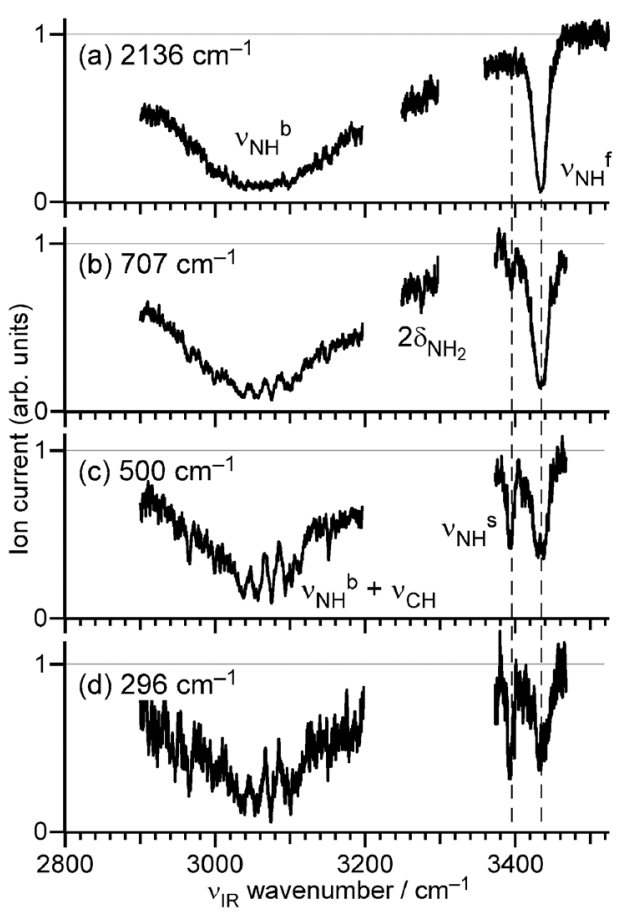

Fig. 6 Excess energy dependence of the IR spectrum of $4 A B N^{+}-W$ ionized via the $\mathrm{S}_{1} \mathrm{O}^{\circ}$ origin band of $4 \mathrm{ABN}-\mathrm{W}(\mathrm{CN})$. The ionization excess energies are indicated in each panel.

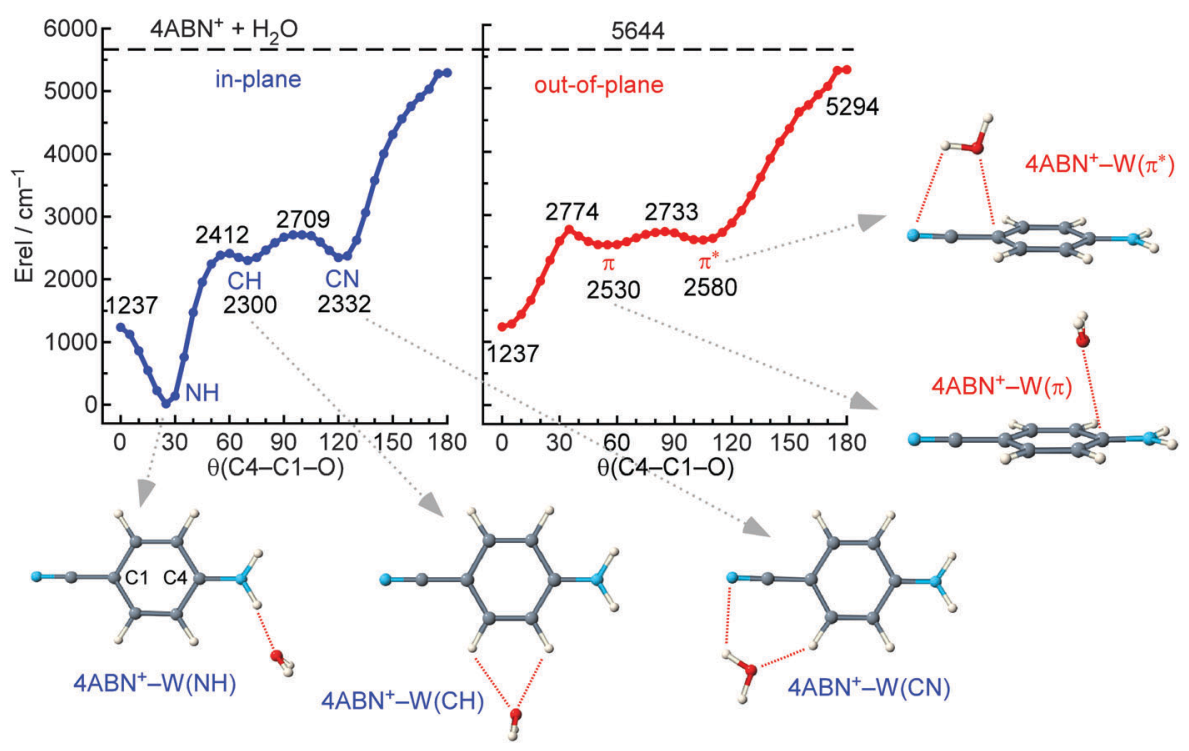

Fig. 5 Potential for $4 \mathrm{ABN}^{+}-\mathrm{H}_{2} \mathrm{O}$ and structures of the minima along the minimum energy paths (M06-2X/aug-cc-pVTZ) for water moving around the $4 \mathrm{ABN}^{+}$cation in the molecular plane (left) and in the plane perpendicular to it (right). ${ }^{44} \mathrm{All} E_{\text {rel }}$ values $\left(\mathrm{cm}^{-1}\right)$ are given with respect to the energy of the $\mathrm{NH}$-bound global minimum $\left(E_{\text {rel }}=0\right) . E_{\text {rel }}=5644 \mathrm{~cm}^{-1}$ corresponds to the dissociation limit into $4 \mathrm{ABN}^{+}$and water (dashed line). 
reasonable because the water migration lifetime is $\sim 17 \mathrm{ps}$, and then the migration reaction must be finished after the $50 \mathrm{~ns}$ delay.

Significantly, the spectral features change when the ionization excess energy was reduced to $707 \mathrm{~cm}^{-1}$ (Fig. 6(b)). Besides $\nu_{\mathrm{NH}}^{\mathrm{f}}$ and $\nu_{\mathrm{NH}}^{\mathrm{b}}$, the weak $\nu_{\mathrm{NH}}^{\mathrm{s}}$ band characteristic of a free amino group is clearly observed although the spectrum was measured at 50 ns delay. When the excess energy is suppressed further down to $500 \mathrm{~cm}^{-1}$ (Fig. 6(c)) and $296 \mathrm{~cm}^{-1}$ (Fig. 6(d)), this spectral signature of the free amino group of $4 \mathrm{ABN}^{+}-\mathrm{W}$ increases in relative intensity and becomes almost comparable to the strength of the $\nu_{\mathrm{NH}}^{\mathrm{f}}$ band. This observation means that some of the nascent $4 \mathrm{ABN}^{+}-\mathrm{W}(\mathrm{CN})$ population does not reach the $\mathrm{NH}$-bound reaction product $\mathrm{P}^{+}$because their internal energy is not high enough to surmount the reaction barrier. It cannot be decided whether these cations are trapped at the $4 \mathrm{ABN}^{+}-\mathrm{W}(\mathrm{CN})$ local minimum or at one of the other local minima along the $\mathrm{CN} \rightarrow \mathrm{NH}_{2}$ reaction pathway(s) such as CH-bound or $\pi^{(*)}$-bound structures (Fig. 5). The sharp structures between $2900-3200 \mathrm{~cm}^{-1}$ appearing on top of the broad $\nu_{\mathrm{NH}}^{\mathrm{b}}$ band in the low excess energy spectra may be explained as follows. These bands are expected to be $\nu_{\mathrm{CH}}$ fundamentals from their positions, although their IR intensities are calculated to be weak (there may also be contributions from overtone and combination bands coupled to $\nu_{\mathrm{NH}}^{\mathrm{b}}$ ). The lower $\mathrm{CN} \rightarrow \mathrm{NH}_{2}$ reaction yield strongly reduces the intensity of $\nu_{\mathrm{NH}}^{\mathrm{b}}$, and the buried weak transitions start to appear under small excess energy conditions. The distribution of the internal energy is determined by the Franck-Condon factor between the $\mathrm{S}_{1}$ origin and the $\mathrm{D}_{0}$ state of the $4 \mathrm{ABN}^{+}-\mathrm{W}(\mathrm{CN})$ structure. For increasing ionization excess energy, more and more vibrational states in the cation become accessible, which increases the population of nascent $4 \mathrm{ABN}^{+}-\mathrm{W}(\mathrm{CN})$ states lying above the migration barrier. As a consequence, the intensity of the $\nu_{\mathrm{NH}}^{\mathrm{f}}$ and $\nu_{\mathrm{NH}}^{\mathrm{b}}$ bands of the final $\mathrm{P}^{+}$reaction product increases with respect to the small but nonvanishing $\nu_{\mathrm{NH}}^{\mathrm{s}}$ signal of the $\mathrm{AF}^{+}$population not reaching the $\mathrm{P}^{+}$state. At a relatively high excess energy of $2136 \mathrm{~cm}^{-1}$, the fraction of the $\mathrm{AF}^{+}$product is very small and almost invisible in the IR spectrum. For such high energies, the $\mathrm{CN} \rightarrow \mathrm{NH}_{2}$ reaction yield approaches unity (as observed in the ps TRIR spectra for an ionization excess energy of $1238 \mathrm{~cm}^{-1}$ ). Since the signatures of both $\mathrm{AF}^{+}$and $\mathrm{P}^{+}$coexist already at an excess energy of $296 \mathrm{~cm}^{-1}$, the effective barrier for the water migration is lower than $296 \mathrm{~cm}^{-1}$ for the nascent $4 \mathrm{ABN}^{+}-\mathrm{W}(\mathrm{CN})$ population, which is consistent with the barrier of $377 \mathrm{~cm}^{-1}$ estimated for cold $4 \mathrm{ABN}^{+}-\mathrm{W}(\mathrm{CN})$ from the M06-2X calculations (Fig. 5). This phenomenon that the reduction of the ionization excess energy may stop an isomerization reaction has been observed earlier for the case of the ionization-induced $\mathrm{OH} \rightarrow \mathrm{NH}_{2}$ migration in 4-aminophenol- $\mathrm{H}_{2} \mathrm{O}{ }^{48}$

Fig. 5 reproduces the salient parts of the potential energy surface of $4 \mathrm{ABN}^{+}-\mathrm{W}$ calculated at the M06-2X/aug-cc-pVTZ level, namely the minimum energy paths for water migration around $\mathrm{ABN}^{+}$in the aromatic plane or in the perpendicular plane. ${ }^{44}$ This potential reveals a strongly bound $4 \mathrm{ABN}^{+}-\mathrm{W}(\mathrm{NH})$ global minimum with a dissociation energy of $D_{\mathrm{e}}=5644 \mathrm{~cm}^{-1}$ and several less stable local minima along the in-plane and out-of-plane paths, including the CN-bound, $\mathrm{CH}$-bound, and $\pi^{(*)}$-bound structures (for details of their structural and vibrational properties, we refer to ref. 44). Most relevant for the current study are the facts that all local minima along the in-plane and out-of-plane pathways have similar relative energies separated by low barriers. Thus, ionization of $4 \mathrm{ABN}-\mathrm{W}(\mathrm{CN})$ reaches $\mathrm{FC}^{+}$states close to the $4 \mathrm{ABN}^{+}-\mathrm{W}(\mathrm{CN})$ minimum and allows for isomerization toward the two equivalent and deep $4 \mathrm{ABN}^{+}-\mathrm{W}(\mathrm{NH})$ global minima via low reaction barriers both along in-plane and out-of-plane pathways. These competing paths are not considered in the current simple rate constant model. Clearly, sophisticated MD simulations are required to analyse the details of the competing reaction pathways and their rate constants. Once the $4 \mathrm{ABN}^{+}-\mathrm{W}(\mathrm{CN})$ isomer has reached the strongly bound $4 \mathrm{ABN}^{+}-\mathrm{W}(\mathrm{NH})$ global minimum, fast IVR removes internal energy out of the reaction coordinate and readily traps water in that minimum, thereby preventing any back reaction to the much less stable local minima. The calculated potential predicts an exothermicity of $2300 \mathrm{~cm}^{-1}$ for the $\mathrm{CN} \rightarrow \mathrm{NH}_{2}$ forward reaction and a high and steep barrier of $2400 \mathrm{~cm}^{-1}$ for the $\mathrm{NH}_{2} \rightarrow \mathrm{CN}$ back reaction. Hence, removal of only a small amount of internal energy from the reaction coordinate via IVR is required to trap water in the deep double minimum potential of the two equivalent $\mathrm{NH}$ sites of the amino group (separated by a barrier of $1237 \mathrm{~cm}^{-1}$ ).

\section{Conclusions}

In summary, the time constant for the dynamics of water migration around the aromatic phenyl ring in $4 \mathrm{ABN}^{+}-\mathrm{W}$ is measured as about 17 ps using ps TRIR spectroscopy. The migration lifetime of $17 \mathrm{ps}$ for the $\mathrm{CN} \rightarrow \mathrm{NH}_{2}$ forward reaction is 3-4 times slower than the $\mathrm{CO} \rightarrow \mathrm{NH}$ reaction in $\mathrm{AA}-\mathrm{W},{ }^{27}$ and the slow migration is rationalized by the difference of the internal energy available from the employed photoionization excess energy. The reaction proceeds with essentially $100 \%$ yield at an ionization excess energy of $1238 \mathrm{~cm}^{-1}$ and the $\mathrm{NH}_{2} \rightarrow \mathrm{CN}$ back reaction is efficiently quenched by rapid IVR of the deep $4 \mathrm{ABN}^{+}-\mathrm{W}(\mathrm{NH})$ global minimum. However, some finite population of nascent $4 \mathrm{ABN}^{+}-\mathrm{W}(\mathrm{CN})$ clusters do not reach the final $\mathrm{NH}$-bound reaction product when the ionization excess energy is reduced, and the $4 \mathrm{ABN}^{+}-\mathrm{W}$ clusters are trapped in the shallow $4 \mathrm{ABN}^{+}-\mathrm{W}(\mathrm{CN})$ local minimum close to the $\mathrm{FC}^{+}$ state and/or in one of the shallow $\mathrm{CH}$ or $\pi^{(*)}$ local minima along the in-plane or out-of-plane isomerization pathways. Although the present experiment provides an initial view on the dynamics of this solvent rearrangement reaction, its preliminary analysis using simple classical rate equations leaves several important questions to be solved in upcoming MD simulations, such as the competition between the various possible reaction pathways and their branching ratios, the appearance of reaction intermediates along these pathways, and the dependence of these parameters on the internal energy available for the reaction. The experiments will be extended along two lines. First, the expected strong dependence of the properties of the reaction on the ionization excess energy will be characterized, particularly at the lowest 
possible excess energy when the reaction is nearly quenched. The second extension is toward larger $4 \mathrm{ABN}-\mathrm{W}_{n}$ clusters $^{44}$ to probe the dynamics of the $\mathrm{H}$-bonded solvent network around the solute at the molecular level, thereby approaching more realistic solute-solvent systems.

\section{Acknowledgements}

This work was supported in part by KAKENHI on innovative area (2503) and the Cooperative Research Program of the "Network Joint Research Center for Materials and Devices" from the Ministry of Education, Culture, Sports, Science and Technology (MEXT), Japan, the Core-to-Core Program 22003 from the Japan Society for the Promotion of Science (JSPS), and the Deutsche Forschungsgemeinschaft (DFG DO 729/4, ENPMI-1236).

\section{References}

1 B. Bagchi, Annu. Rev. Phys. Chem., 1989, 40, 115-141.

2 W. P. de Boeij, M. S. Pshenichnikov and D. A. Wiersma, Annu. Rev. Phys. Chem., 1998, 49, 99-123.

3 G. R. Fleming and M. H. Cho, Annu. Rev. Phys. Chem., 1996, 47, 109-134.

4 H. J. Bakker and J. L. Skinner, Chem. Rev., 2010, 110, 1498-1517.

5 N. Nandi, K. Bhattacharyya and B. Bagchi, Chem. Rev., 2000, 100, 2013-2045.

6 R. Jimenez, G. R. Fleming, P. V. Kumar and M. Maroncelli, Nature, 1994, 369, 471-473.

7 E. W. Castner, G. R. Fleming and B. Bagchi, J. Chem. Phys., 1988, 89, 3519-3534.

8 M. S. Cheung, A. E. Garcia and J. N. Onuchic, Proc. Natl. Acad. Sci. U. S. A., 2002, 99, 685-690.

9 G. A. Papoian, J. Ulander, M. P. Eastwood, Z. LutheySchulten and P. G. Wolynes, Proc. Natl. Acad. Sci. U. S. A., 2004, 101, 3352-3357.

10 R. H. Zhou, Proc. Natl. Acad. Sci. U. S. A., 2003, 100, 13280-13285.

11 W. Y. Lu, W. H. Qiu, J. Kim, O. Okobiah, H. X. Hu, G. W. Gokel and D. P. Zhong, Chem. Phys. Lett., 2004, 394, 415-422.

12 K. Suzuki, M. Kawano and M. Fujita, Angew. Chem., Int. Ed., 2007, 46, 2819-2822.

13 C. L. Shen and R. M. Murphy, Biophys. J., 1995, 69, 640-651.

14 A. N. Rissanou, E. Georgilis, E. Kasotaids, A. Mitraki and V. Harmandaris, J. Phys. Chem. B, 2013, 117, 3962-3975.

15 J. He, X. L. Huang, Y. C. Li, Y. J. Liu, T. Babu, M. A. Aronova, S. J. Wang, Z. Y. Lu, X. Y. Chen and Z. H. Nie, J. Am. Chem. Soc., 2013, 135, 7974-7984.

16 N. Sarkar, K. Das, A. Datta, S. Das and K. Bhattacharyya, J. Phys. Chem., 1996, 100, 10523-10527.

17 S. M. Bhattacharyya, Z. G. Wang and A. H. Zewail, J. Phys. Chem. B, 2003, 107, 13218-13228.

18 L. Nilsson and B. Halle, Proc. Natl. Acad. Sci. U. S. A., 2005, 102, 13867-13872.
19 A. Stolow, A. E. Bragg and D. M. Neumark, Chem. Rev., 2004, 104, 1719-1757.

20 Q. Zhong and J. A. W. Castleman, Chem. Rev., 2000, 100, 4039-4057.

21 M. Fujii and O. Dopfer, Int. Rev. Phys. Chem., 2012, 31, 131-173.

22 O. Dopfer, Z. Phys. Chem., 2005, 219, 125-168.

23 T. S. Zwier, Annu. Rev. Phys. Chem., 1996, 47, 205-241.

24 B. Brutschy, Chem. Rev., 2000, 100, 3891-3920.

25 T. Ebata, A. Fujii and N. Mikami, Int. Rev. Phys. Chem., 1998, 17, 331-361.

26 A. B. Wolk, C. M. Leavitt, E. Garand and M. A. Johnson, Acc. Chem. Res., 2014, 47, 202-210.

27 K. Tanabe, M. Miyazaki, M. Schmies, A. Patzer, M. Schütz, H. Sekiya, M. Sakai, O. Dopfer and M. Fujii, Angew. Chem., Int. Ed., 2012, 51, 6604-6607.

28 M. Miyazaki, A. Takeda, S. Ishiuchi, M. Sakai, O. Dopfer and M. Fujii, Phys. Chem. Chem. Phys., 2011, 13, 2744-2747.

29 S. Ishiuchi, M. Miyazaki, M. Sakai, M. Fujii, M. Schmies and O. Dopfer, Phys. Chem. Chem. Phys., 2011, 13, 2409-2416.

30 S. Ishiuchi, M. Sakai, Y. Tsuchida, A. Takeda, Y. Kawashima, O. Dopfer, K. Müller-Dethlefs and M. Fujii, J. Chem. Phys., 2007, 127, 114307.

31 S. Ishiuchi, M. Sakai, K. Daigoku, K. Hashimoto and M. Fujii, J. Chem. Phys., 2007, 127, 234304.

32 S. Ishiuchi, M. Sakai, Y. Tsuchida, A. Takeda, Y. Kawashima, M. Fujii, O. Dopfer and K. Müller-Dethlefs, Angew. Chem., Int. Ed., 2005, 44, 6149-6151.

33 S. Ishiuchi, M. Sakai, K. Daigoku, T. Ueda, T. Yamanaka, K. Hashimoto and M. Fujii, Chem. Phys. Lett., 2001, 347, 87-92.

34 M. Wohlgemuth, M. Miyazaki, M. Weiler, M. Sakai, O. Dopfer, M. Fujii and R. Mitrić, Angew. Chem., Int. Ed., 2014, 53, 14601-14604.

35 E. M. Gibson, A. C. Jones and D. Phillips, Chem. Phys. Lett., 1988, 146, 270-274.

36 E. M. Gibson, A. C. Jones, W. G. Bouwman, D. Phillips and J. Sandell, J. Phys. Chem., 1988, 92, 5449-5455.

37 E. Alejandro, J. A. Fernández and F. Castaño, Chem. Phys. Lett., 2002, 353, 195-203.

38 E. Alejandro, C. Landajo, A. Longarte, J. A. Fernández and F. Castaño, J. Chem. Phys., 2003, 119, 9513-9522.

39 K. Sakota, N. Yamamoto, K. Ohashi, M. Saeki, S. Ishiuchi, M. Sakai, M. Fujii and H. Sekiya, Chem. Phys., 2002, 283, 209-219.

40 K. Sakota, N. Yamamoto, K. Ohashi, H. Sekiya, M. Saeki, S. Ishiuchi, M. Sakai and M. Fujii, Chem. Phys. Lett., 2001, 341, 70-76.

41 K. Sakota, N. Yamamoto, K. Ohashi, M. Saeki, S. Ishiuchi, M. Sakai, M. Fujii and H. Sekiya, Phys. Chem. Chem. Phys., 2003, 5, 1775-1779.

42 M. A. Lee, S. H. Nam, H. S. Park, N. R. Cheong, S. Ryu, J. K. Song and S. M. Park, Bull. Korean Chem. Soc., 2008, 29, 2109-2113.

43 T. Nakamura, M. Schmies, A. Patzer, M. Miyazaki, S. Ishiuchi, M. Weiler, O. Dopfer and M. Fujii, Chem. - Eur. J., 2014, 20, 2031-2039. 
44 M. Schmies, M. Miyazaki, M. Fujii and O. Dopfer, J. Chem. Phys., 2014, 141, 214301.

45 M. Miyazaki, A. Fujii, T. Ebata and N. Mikami, Chem. Phys. Lett., 2001, 349, 431-436.

46 N. Solca and O. Dopfer, Chem. Phys. Lett., 2001, 347, 59-64.

47 H. Tachikawa and M. Igarashi, J. Phys. Chem. A, 1998, 102, 8648-8656.

48 M. Gerhards, A. Jansen, C. Unterberg and A. Gerlach, J. Chem. Phys., 2005, 123, 074320.

49 T. Ikeda, K. Sakota, Y. Kawashima, Y. Shimazaki and H. Sekiya, J. Phys. Chem. A, 2012, 116, 3816-3823.

50 K. Sakota, Y. Shimazaki and H. Sekiya, Phys. Chem. Chem. Phys., 2011, 13, 6411-6415.

51 H. Tachikawa, M. Igarashi and T. Ishibashi, J. Phys. Chem. A, 2003, 107, 7505-7513.

52 K. Sakota, S. Harada, Y. Shimazaki and H. Sekiya, J. Phys. Chem. A, 2011, 115, 626-630.

53 M. Weiler, T. Nakamura, H. Sekiya, O. Dopfer, M. Miyazaki and M. Fujii, ChemPhysChem, 2012, 13, 3875-3881.
54 K. Sakota, Y. Kouno, S. Harada, M. Miyazaki, M. Fujii and H. Sekiya, J. Chem. Phys., 2012, 137, 224311.

55 M. Schütz, K. Sakota, R. Moritz, M. Schmies, T. Ikeda, H. Sekiya and O. Dopfer, J. Phys. Chem. A, 2015, 119, 10035-10051.

56 H. M. Kim, K. Y. Han, J. Park, G. S. Kim and S. K. Kim, J. Chem. Phys., 2008, 128, 041104.

57 T. Kobayashi and O. Kajimoto, J. Chem. Phys., 1987, 86, 1118-1124.

58 T. Kobayashi, K. Honma, O. Kajimoto and S. Tsuchiya, J. Chem. Phys., 1987, 86, 1111-1117.

59 T. Nakamura, M. Miyazaki, S. Ishiuchi, M. Weiler, M. Schmies, O. Dopfer and M. Fujii, ChemPhysChem, 2013, 14, 741-745.

60 T. Omi, H. Shitomi, N. Sekiya, K. Takazawa and M. Fujii, Chem. Phys. Lett., 1996, 252, 287-293.

61 M. Schmies, A. Patzer, S. Kruppe, M. Miyazaki, S. Ishiuchi, M. Fujii and O. Dopfer, ChemPhysChem, 2013, 14, 728-740. 62 S. Ullrich and K. Müller-Dethlefs, J. Phys. Chem. A, 2002, 106, 9188-9195.

63 M. Schmies, PhD thesis, Technische Universität Berlin, 2015. 